\title{
Validación del Método de Microondas para Determinar Humedad en Ñame Espino (Dioscorea rotundata Poir)
}

\author{
Carlos Garcia-Mogollon ${ }^{(1) \star}$, Armando Alvis-Bermudez ${ }^{(2)}$, Saúl Dussán-Sarria ${ }^{(3)}$ \\ (1) Facultad de Ingeniería, Grupo PADES, Universidad de Sucre. Sincelejo, Sucre, Colombia. \\ (e-mail: carlos.garcia@unisucre.edu.co) \\ (2) Facultad de Ingenierías, Grupo GIPAVE, Universidad de Córdoba, Cereté, Córdoba, Colombia. \\ (3) Fac.de Ingeniería y Administración, Depto. de Ingeniería, Univ. Nacional de Colombia. Palmira, \\ Colombia.
}

${ }^{*}$ Autor a quien debe ser dirigida la correspondencia.

Recibido Ago. 2, 2016; Aceptado Sep. 12, 2016; Versión final Nov. 28, 2016, Publicado Abr. 2017

\begin{abstract}
Resumen
El objetivo de esta investigación fue validar el método de microondas para la determinación de humedad en ñame espino (Dioscorea rotundata Poir). Las muestras de ñame fueron lavadas con agua potable, peladas, cortadas en rodajas de $1.0 \mathrm{~cm}$ de diámetro por $0.6 \mathrm{~cm}$ de espesor y fueron pesadas en submuestras de $2 \mathrm{~g} \mathrm{a} 5 \mathrm{~g}, 5 \mathrm{~g}$ a $7 \mathrm{~g}$ y $7 \mathrm{~g}$ a $10 \mathrm{~g}$. Se utilizó el método 925.09 AOAC para determinar la humedad de referencia y el método de microondas aplicando potencias de $280 \mathrm{~W}, 560 \mathrm{~W}, 700 \mathrm{~W}, 840 \mathrm{~W}$. Se tomó el peso inicial de cada muestra y el peso final al cabo de lotes de secado en el microondas de 1 minuto de duración y hasta llegar a peso constante de la muestra. Para la validación del método se tuvo en cuenta la exactitud, precisión, la relación de Horwitz, repetibilidad y reproducibilidad. El tratamiento a $700 \mathrm{~W}, 2 \mathrm{~g}$ a $5 \mathrm{~g}$ y 6 min cumple con los parámetros de validación precisión, recuperación, repetibilidad y reproducibilidad para determinar la humedad en ñame espino.
\end{abstract}

Palabras claves: ñame; contenido de humedad; método del microondas; Dioscorea rotundata Poir

\section{Validation of the method of microwave for determining moisture in yam hawthorn (Dioscorea rotundata Poir)}

\begin{abstract}
The objective of this research was to validate the method for the determination of moisture in hawthorn yam (Dioscorea rotundata Poir) by application of microwave. Yam samples were washed with tap water, peeled and cut into sliced of $1.0 \mathrm{~cm}$ in diameter and $0.6 \mathrm{~cm}$ thick, were weighed in samples of 2 to $5 \mathrm{~g}, 5$ to $7 \mathrm{~g}$ and 7 to $10 \mathrm{~g}$. The method $925.09 \mathrm{AOAC}$ was used to determine the reference humidity and the microwave method at powers of $280 \mathrm{~W}, 560 \mathrm{~W}, 700 \mathrm{~W}, 840 \mathrm{~W}$. The initial and the final weights were recorded for each process I the microwave (1 minute duration) were recorded. To validate the method the following factors were considered: accuracy, precision, the Horwitz ratio, repeatability and reproducibility. The treatment at $700 \mathrm{~W}, 2 \mathrm{~g}$ a $5 \mathrm{~g}$ y 6 min fulfills the necessary parameters of validation for ñame espino.
\end{abstract}




\section{INTRODUCCIÓN}

El ñame (Dioscorea rotundata Poir) es un tubérculo utilizado como alimento básico en muchas regiones tropicales, principalmente en el occidente de África, Asia, el Pacifico y el Caribe (Martínez y Ortiz, 2009). El Caribe colombiano representa más del $90 \%$ de la producción nacional de ñame. En Colombia se cosecharon alrededor de 314.991 ton en 2013, con un rendimiento de 10,7 ton por hectárea sembrada (Agronet, 2015), siendo los departamentos con mayor participación Bolívar, Córdoba y Sucre. En la región Caribe colombiana, las preparaciones del ñame abarcan productos salados y dulces, ejemplo de ello es el tradicional mote de queso y el dulce de ñame, usualmente elaborado en época de Semana Santa (Reina, 2012). Al deshidratarse el ñame bajo la forma de harina puede ser utilizado en la elaboración de productos tales como sopas, galletas, panes, bebidas y pudines (Espinosa, 2009).

Para determinar la humedad en un alimento se utilizan métodos como el uso de luz infrarroja (Mantanus et al., 2009), resonancia magnética nuclear (Sorland et al., 2004) y métodos volumétricos como el de Karl Fischer (Gallina et al., 2010). La radiación electromagnética de alta frecuencia, en el proceso de secado de productos agrícolas utilizando microondas presenta rápido aumento de la temperatura en las muestras y puede ocasionar incineración del material eliminándose almidón, grasas, proteínas además de agua, lo que implica una fuente de error para el método. Por estas razones es recomendado determinar el tiempo de exposición máximo de la muestra a la radiación en varias potencias para evitar incinerar el material (Casada et al., 1983). El componente más importante en esta interacción son las moléculas de agua del alimento (Berk, 2009) y productos como el ñame, yuca y plátano son de alta humedad facilitando la aplicación de microondas.

Se han realizado estudios para determinar la humedad utilizando la energía del horno microondas en granos (Gorakhurwalla et al., 1975), productos agrícolas (Click y Baker, 1980), semilla de maíz (Kraszewski et al., 1991), lácteos (Kress-Rogers y Kent, 1987), materiales en polvo y alimentos (Nyfors, 2000). Además, otros estudios en donde se comparan la determinación de humedad por métodos convencionales y microondas, concluyéndose que no se encontró diferencia entre las técnicas aplicadas y sugiriendo el empleo del horno microondas por menor tiempo de exposición, eficiencia y practicidad en la determinación del contenido de humedad (García et al., 2012, Tirado et al., 2015); además, de su utilidad en la industria de alimentos por presentar menor consumo de energía, mayor comodidad y rapidez y, por consiguiente, mayor economía operacional. Por otra parte, la utilización de la energía del horno microondas há sido relatada como viable em la reducción microbiana de alimentos, mejorando la calidad microbiológica y prolongando la vida de anaquel (Aymerich et al., 2008). El objetivo de este estudio fue el de validar el método para la determinación de humedad en ñame espino (Dioscorea rotundata) mediante la aplicación de energía de microondas. El objetivo de este estudio fue el de validar el método para la determinación de humedad en ñame espino (Dioscorea rotundata) mediante la aplicación de energía de microondas.

\section{MATERIALES Y MÉTODOS}

Se describe la determinación de humedad, luego se explica el método de microondas, se presenta el diseño experimental y finalmente se explica la validación de los datos.

\section{Local y materia prima}

El experimento se realizó en el Laboratorio GIPAVE de la Universidad de Córdoba, en la ciudad de Monteria, Colombia. La materia prima correspondió a frutos de ñame espino el cual fue adquirido en el mercado local con madurez fisiológica superior al $90 \%$, es decir, el tubérculo estaba completamente de color marrón o negruzco (Ávila-Tordecilla, 2013).

\section{Determinación de humedad de referencia}

El contenido de humedad de referencia $\left(\% \mathrm{H}_{\text {ret }}\right)$ fue determinado a través del método convencional 925.09 AOAC en la cual las muestras de ñame (Dioscorea rotundata) provenientes de la ciudad de Montería, Córdoba, Colombia. Fueron lavadas con agua potable, peladas y rebanadas formado chips de $1.0 \mathrm{~cm}$ de diámetro y $0.6 \mathrm{~cm}$ de espesor. Para su homogenización se cuartearon, porcionaron en unidades de 2 a $5 \mathrm{~g}$ y secaron en estufa (Technicook modelo MINICONV) con aire forzado de $100-105^{\circ} \mathrm{C}$ de $1 \mathrm{a} 5 \mathrm{~h}$ hasta peso constante al tercer decimal. Posteriormente se colocaron $20 \mathrm{~min}$ en el desecador. El porcentaje de humedad (ecuación 1) en base húmeda se calculó con el peso inicial de la muestra (Pi) y el peso final (Pf). Se utilizó la balanza digital modelo EW-BAL 1.3 de la marca Heraeus con sensibilidad de $0,001 \mathrm{~g}$. 
Se utilizó un horno microondas doméstico de marca Haceb HM-1.1 con una frecuencia de $2450 \mathrm{MHz}$ y dimensiones internas de $354 \mathrm{~mm} \times 228 \mathrm{~mm} \times 373 \mathrm{~mm}$. Las muestras se acondicionaron igual al procedimiento de determinación de humedad de referencia. Para cada potencia aplicada ( $280 \mathrm{~W}, 560 \mathrm{~W}, 700 \mathrm{~W}$ y $840 \mathrm{~W}$ ) y por cada tamaño de muestra se procedió según el diseño experimental. El horno microondas poseía plato giratorio y el registro de la pérdida de peso de cada muestra de ñame se realizó cada 1 minuto hasta obtener un valor próximo en la segunda casa decimal del contenido de humedad de referencia y así obtener un tiempo de referencia; el cual, fue usado como punto de partida para realizar el proceso de validación.. Posterior a la aplicación del tratamiento (potencia - tiempo de referencia) a cada muestra fue retirada del microondas, colocada en un desecador durante $20 \mathrm{~min}$ y pesada. El contenido de humedad se expresa tal como la $\mathrm{H}_{\text {ref. }}$

\section{Diseño experimental}

Se aplicó un diseño experimental completamente al azar con 12 tratamientos en arreglo factorial $3 \times 4$ con los factores: rango peso de la muestra (2 a $5 \mathrm{~g}, 5 \mathrm{a} 7 \mathrm{~g}$ y 7 a $10 \mathrm{~g}$ ) y potencia $(280 \mathrm{~W}, 560 \mathrm{~W}, 700 \mathrm{~W}$ y $840 \mathrm{~W})$. Para cada periodo de exposición se realizaron 5 réplicas y utilizaron 8 muestras por ensayo. A los tratamientos en cada potencia que no presentaron diferencia significativa con la humedad de referencia (Href) se les determinó la prueba bilateral de comparación de medias a un nivel de confianza del 95\%. A los tiempos de los tratamientos que resultaron iguales se confirmaron con una prueba de t-Student para dos muestras independientes. Los datos se procesaron haciendo uso de la hoja de cálculo Microsoft Excel $\circledast$ versión 2010.

\section{Validación}

Para validar el método de microondas se usan los parámetros analíticos exactitud, precisión, repetibilidad y límite de recuperación (R) aceptable de 98 a 101\%. Acorde a la Categoría I correspondiente a métodos que cuantifican un componente mayoritario en el alimento. La exactitud se determinó como el Error (ecuación 1) entre la humedad del método evaluación y la humedad de referencia.

$\%$ Error $=\frac{\% H-\% H_{r e f}}{\% H_{r e f}}$

Para establecer la precisión esperada se usó la ecuación de Horwitz (ecuación 2) acorde a los parámetros de procedimientos de Validación de Laboratorio Único de la AOAC (SLV: Single Laboratory Validation) (Hall, 2008).

HorwitzRSD $D_{r}=2 C^{-0,1505}$

Donde $\mathrm{C}$ es la concentración del analito en la matriz (contenido de agua), expresado con dos decimales. La ecuación de Horwitz se utilizó para la determinación de la desviación estándar en las diferentes repeticiones tanto para los datos del contenido de humedad utilizando el horno microondas (RSDr) como para los datos del contenido de humedad de referencia (PRSDr). Y se determinó la aceptabilidad de la precisión con la relación de Horwitz (ecuación 3). Valores de HorRat de 0,3 a 1,3 son considerados aceptables para SLV (Hall, 2008).

HorRat $=\frac{\text { RSD }_{\mathrm{r}}}{\text { PRSD }_{\mathrm{r}}}$

Para el estudio de repetibilidad se establecieron los valores de los parámetros: el número de réplicas hechas cada día ( $r$ igual a 8 , el número de las muestras utilizadas (n) igual a 3 y el número de días que se realizó el estudio (d) igual a 3 . Teniendo en cuenta las especificaciones que $n$ debe ser de al menos $2, d$ debe ser de al menos 2 y el producto $r x d$ nunca debe ser menor que 10. A los valores de humedad obtenidos se determinó la media, desviación estándar y coeficiente de variación.

\section{RESULTADOS Y DISCUSIÓN}

La composición de los chips de ñame presenta una humedad de referencia de $61,60 \pm 0,03 \%$ y coeficiente de variación (CV) de 2,12\%. García et al. (2012) evaluaron el método de secado en microondas obteniendo un contenido de humedad de $65,55 \pm 0,0057 \%$ para el ñame y CV menores al $3 \%$, definiéndolos como valores permitidos en el reporte de humedad; Bhandari et al. (2003) caracterizaron varias especies de ñame 
encontrándose en la variedad Rotundata humedades de 65,8\% y Romero y Jiménez (2014) evaluaron el secado del ñame en microondas encontrando humedades de $65,73 \%$.

Validación del método con microondas

La utilización de microondas en todas las potencias y pesos de muestras evaluadas redujo sustancialmente el tiempo de secado requerido para obtener el valor de humedad de referencia en las muestras de ñame cuando se compara al horno de secado convencional.

Tabla 1: Contenido de humedad y tiempo de secado para el tratamiento de microondas

\begin{tabular}{|c|c|c|l|}
\hline Potencia $(W)$ & Peso $(g)$ & Tiempo $(\mathrm{min})$ & \multicolumn{1}{|c|}{ Humedad (\%) } \\
\hline 280 & 2 a 5 & 20 & $60,86 \pm 0,02 \mathrm{cde}$ \\
\hline 280 & 5 a 7 & 36 & $58,03 \pm 0,03 \mathrm{bc}$ \\
\hline 280 & 7 a 10 & 42 & $61,64 \pm 0,043 \mathrm{e}$ \\
\hline 560 & 2 a 5 & 7 & $59,87 \pm 0,01 \mathrm{bcd}$ \\
\hline 560 & 5 a 7 & 8 & $59,52 \pm 0,01 \mathrm{bcd}$ \\
\hline 560 & 7 a 10 & 8 & $58,23 \pm 0,01 \mathrm{c}$ \\
\hline 700 & 2 a 5 & 6 & $61,01 \pm 0,01 \mathrm{de}$ \\
\hline 700 & 5 a 7 & 6 & $61,12 \pm 0,01 \mathrm{de}$ \\
\hline 700 & 7 a 10 & 6 & $59,29 \pm 0,01 \mathrm{bcd}$ \\
\hline 840 & 2 a 5 & 6 & $54,40 \pm 0,04 \mathrm{~b}$ \\
\hline 840 & 5 a 7 & 6 & $52,55 \pm 0,01 \mathrm{~b}$ \\
\hline 840 & 7 a 10 & 6 & $52,57 \pm 0,04 \mathrm{a}$ \\
\hline
\end{tabular}

A medida que aumenta la potencia del microondas, se reduce el tiempo requerido de secado, excepto para $700 \mathrm{~W}$ y $840 \mathrm{~W}$ donde los tiempos fueron los mismos. Sin embargo, para la potencia de microondas de $840 \mathrm{~W}$ se observa un deterioro por quemadura de los chips de ñame que se ocasionó por las microondas al trabajar con una potencia muy alta. Según Casada et al. (1983) esta incineración de los chips pudo ocasionar la eliminación de almidón, grasas y proteínas, además de agua, lo que conduce a un error para la determinación de la humedad. El contenido de humedad en las muestras no presentaron diferencias significadas $(p<0,05)$ en la prueba t-Student al compararlas con la Href en los tratamientos a $280 \mathrm{~W}$ y $700 \mathrm{~W}$. Al observar estos resultados se analiza que es menos conveniente la masa de $7 \mathrm{a} 10 \mathrm{~g}$, dado que se necesitó mucho más tiempo para alcanzar la humedad de referencia y lo que se busca es disminuir los periodos de determinación de humedad que se presentan con el método convencional.

La precisión de los tratamientos se evalúa teniendo en cuenta el error estándar y el CV (Tabla 2). El error estándar varió entre $0,4 \%$ hasta $14,6 \%$, siendo las condiciones de potencia $700 \mathrm{~W}$ con pesos de la muestra de 2 a $5 \mathrm{~g}$ y de $5 \mathrm{a} 7 \mathrm{~g}$ las que indican un menor porcentaje de error en comparación con la Href. Por su parte los valores con mayor error estándar $(11,6$ a $14,6 \%)$ se encuentran en la potencia $840 \mathrm{~W}$ para los tres pesos de muestra utilizadas, evidenciando el efecto del deterioro por quemadura en la determinación. El coeficiente de variación presentó diferencias significativas $(p>0,05)$, representando una mayor variabilidad para los tratamientos a potencias más bajas, mientras que los CV con valores más bajos se encontraron en las potencias de $560 \mathrm{~W}, 700 \mathrm{~W}$ y $840 \mathrm{~W}$, presentándose una menor variación en los tratamientos con las condiciones de $840 \mathrm{~W}$ con peso de 7 a $10 \mathrm{~g}, 560 \mathrm{~W}$ con peso de 7 a $10 \mathrm{~g}$ y $700 \mathrm{~W}$ con peso de 2 a $5 \mathrm{~g}$.

Todos los tratamientos estudiados son válidos ( $\mathrm{CV}<3 \%$ ), excepto el tratamiento a $840 \mathrm{~W}$ con peso de 5 a $7 \mathrm{~g}$ que tiene un valor de CV de 3,3\%. Estudios similares presentó Oetzel et al. (1993) quien comparó varios métodos de secado en alimentos ensilados, registrando en CV de 1,4 y 1,3\% para el método convencional y microondas respectivamente. Los tratamientos que presentaron diferencias significativas $(p>0.05)$ con respecto a la humedad de referencia son los siguientes: $840 \mathrm{~W}$ con pesos de $2 \mathrm{a} 5 \mathrm{~g}, 5$ a $7 \mathrm{~g}$ y $7 \mathrm{a} 10 \mathrm{~g} ; 280 \mathrm{~W}$ con peso de 5 a $7 \mathrm{~g}$ y $560 \mathrm{~W}$ con peso de la muestra de 7 a $10 \mathrm{~g}$. Los tratamientos que no presentaron diferencias significativas $(p<0.05)$ fueron: $700 \mathrm{~W}$ con pesos de 2 a $5 \mathrm{~g}, 5$ a $7 \mathrm{~g}$ y 7 a $10 \mathrm{~g} ; 560 \mathrm{~W}$ con pesos de 2 a $5 \mathrm{~g}$ y 5 a $7 \mathrm{~g}$ y $280 \mathrm{~W}$ con peso de 2 a $5 \mathrm{~g}$. Estudios similares presentaron García et al. (2012) quienes utilizaron el horno microondas en la determinación de contenido de humedad en yuca, ñame y plátano, Gallina et al. (2010) con el método Karl Fischer, Mantanus et al. (2009) con luz infraroja y Sorland et al. (2004) con resonancia magnética nuclear (RMN). 
Tabla 2: Comparación de los diferentes tratamientos con el contenido de humedad de referencia

\begin{tabular}{|c|c|c|c|c|c|}
\hline Potencia (W) & Peso (g) & Tiempo (min) & Humedad (\%) & Error (\%) & CV (\%) \\
\hline 280 & 2 a 5 & 20 & $60,86 \pm 0,02$ & 1,1 & 2,98 \\
\hline 280 & 5 a 7 & 36 & $58,03 \pm 0,02$ & 5,7 & 2,57 \\
\hline 560 & 2 a 5 & 7 & $59,87 \pm 0,01$ & 2,7 & 2,05 \\
\hline 560 & 5 a 7 & 8 & $59,52 \pm 0,01$ & 3,3 & 1,14 \\
\hline 560 & 7 a 10 & 8 & $58,23 \pm 0,01$ & 5,4 & 0,39 \\
\hline 700 & 2 a 5 & 6 & $61,01 \pm 0,01$ & 0,4 & 0,6 \\
\hline 700 & 5 a 7 & 6 & $61,12 \pm 0,01$ & 0,7 & 1,87 \\
\hline 700 & 7 a 10 & 6 & $59,29 \pm 0,01$ & 3,6 & 1,43 \\
\hline 840 & 2 a 5 & 6 & $54,40 \pm 0,04$ & 11,6 & 1,8 \\
\hline 840 & 5 a 7 & 6 & $52,55 \pm 0,01$ & 14,6 & 3,3 \\
\hline 840 & 7 a 10 & 6 & $52,57 \pm 0,02$ & 14,6 & 0,08 \\
\hline
\end{tabular}

A los tratamientos que no presentaron diferencias significativas $(p<0.05)$ se evaluó el porcentaje de recuperación $(\mathrm{R})$ y la precisión esperada según Horwitz por la relación HorRat (Tabla 3). Los límites de recuperación estuvieron entre $96,34 \%$ y $100,44 \%$ y se consideran veraces acorde al rango de $98 \%$ a $101 \%$ establecido por Zumbado (2004). El porcentaje de recuperación (R) para los tratamientos de 560W y el de $700 \mathrm{~W}$ con peso de 7 a $10 \mathrm{~g}$ están por debajo del límite de recuperación, lo que no garantiza la veracidad de la determinación. Estudios similares presentaron García et al. (2012) quienes determinaron humedad en microondas para yuca, ñame y plátano, encontrando que a potencias de $140 \mathrm{~W}$ y $280 \mathrm{~W}$ presentaron valores por fuera del límite de recuperación.

Tabla 3: Porcentaje de recuperación $(\mathrm{R})$ y relación de Horwitz (HorRat)

\begin{tabular}{|c|c|c|c|c|c|c|c|}
\hline $\begin{array}{c}\text { Potencia } \\
(W)\end{array}$ & $\begin{array}{c}\text { Peso } \\
(\mathrm{g})\end{array}$ & $\begin{array}{c}\text { Tiempo } \\
(\mathrm{min})\end{array}$ & Humedad (\%) & $\begin{array}{c}\text { \% Error } \\
\text { estándar }\end{array}$ & CV (\%) & $R(\%)$ & HorRat \\
\hline 280 & 2 a 5 & 20 & $60,86 \pm 0,02$ & 1,1 & 2,98 & 98,90 & 1,002 \\
\hline 560 & 2 a 5 & 7 & $59,87 \pm 0,01$ & 2,7 & 2,05 & 97,29 & 1,004 \\
\hline 560 & 5 a 7 & 8 & $59,52 \pm 0,01$ & 3,3 & 1,14 & 96,72 & 1,005 \\
\hline 700 & 2 a 5 & 6 & $61,01 \pm 0,01$ & 0,4 & 0,60 & 100,44 & 0,999 \\
\hline 700 & 5 a 7 & 6 & $61,12 \pm 0,01$ & 0,7 & 1,87 & 99,32 & 1,001 \\
\hline 700 & 7 a 10 & 6 & $59,29 \pm 0,01$ & 3,6 & 1,43 & 96,34 & 1,006 \\
\hline
\end{tabular}

De acuerdo a los resultados mostrados en la tabla 3 la relación de HorRat varió entre 0,999 y 1,006. Estos valores se encuentran en el rango de 0,3 a 1,3 considerados aceptables por Zumbado (2004) y Duffau et al. (2010) para validar el tratamiento con microondas. De acuerdo al límite de recuperación y la precisión los tratamientos de $560 \mathrm{~W}$ con peso de 2 a $5 \mathrm{~g}$ y 5 a $7 \mathrm{~g}$, 700W con peso de 7 a $10 \mathrm{~g}$ no son aceptables para la validación, del mismo modo el tratamiento de $280 \mathrm{~W}$ con peso de 2 a $5 \mathrm{~g}$ es menos conveniente por el largo periodo de tiempo que se necesita para determinar la humedad. De esta forma los tratamientos de $700 \mathrm{~W}$ con peso de 2 a $5 \mathrm{~g}$ y 5 a $7 \mathrm{~g}$ son los que mejor precisión y recuperación presentaron. En el estudio de repetibilidad y reproducibilidad no se encontraron diferencias significativas $(p<0.05)$ entre los CV de los tratamientos y el de referencia. En el estudio de repetibilidad (Tabla 4) se observa que las muestras con peso de 2 a $5 \mathrm{~g}$ presentaron menores coeficientes de variación en comparación con las muestras de 5 a $7 \mathrm{~g}$.

Tabla 4: Estudio de repetibilidad en la determinación del contenido de humedad con energía del microondas en comparación con el método convencional

\begin{tabular}{|c|c|c|c|c|c|}
\hline $\begin{array}{c}\text { Potencia } \\
(W)\end{array}$ & \multicolumn{2}{|c|}{ Peso $(g)$} & Potencia (W) & \multicolumn{2}{c|}{ Peso $(g)$} \\
\hline 700 & \multicolumn{2}{|c|}{2 a 5} & 700 & \multicolumn{2}{c|}{5 a 7} \\
\hline Día & Humedad (\%) & CV $(\%)$ & Día & Humedad (\%) & CV (\%) \\
\hline 1 & $65,71 \pm 0,01$ & 1,59 & 1 & $66,59 \pm 0,01$ & 1,49 \\
\hline 2 & $59,66 \pm 0,01$ & 1,20 & 2 & $60,52 \pm 0,01$ & 1,63 \\
\hline 3 & $61,40 \pm 0,02$ & 2,95 & 3 & $58,20 \pm 0,02$ & 4,24 \\
\hline
\end{tabular}


El estudio de reproducibilidad en donde seis analistas determinaron la humedad de los chips de ñame a condiciones de $700 \mathrm{~W} / 2$ a $5 \mathrm{~g} / 6 \mathrm{~min}$ y $700 \mathrm{~W} / 5$ a $7 \mathrm{~g} / 6 \mathrm{~min}$ con un coeficiente de variación medio de $2,68 \pm 1,79 \%$ y $1,85 \pm 0,94 \%$ respectivamente. El CV para la mayoría de los analistas fue menor al $3 \%$ indicando que el procedimiento es reproducible independientemente del analista.

\section{CONCLUSIONES}

El método de humedad por microondas puede ser usado de forma precisa para conocer el contenido de humedad en el ñame. La utilización de un horno microondas a 700W de potencia con una muestra de 2 a 5 g durante 6 min resultó el tratamiento más adecuado paras determinar el contenido de humedad en ñame espino.

\section{REFERENCIAS}

Agronet. Estadísticas Agroforestales Consolidado Nacional (2013), https://goo.gl/krJISf. Acceso: 20 de julio (2015)

A.O.A.C: Association of Official Analytical Chemists. Official Methods of Analysis, Methods 925.09: Moisture in cassava - air oven methods, Washington D.C., USA (2005)

Ávila-Tordecilla, M., Manejo Integrado del cultivo del ñame espino (2013).

Aymerich, T., P.A. Picouet, y J.M. Monfort, Decontamination technologies for meat products, Meat Science, 78, 114-129 (2008)

Berk, Z., Food Process Engineering and Technology, Academic Press, USA (2009)

Bhandari, M., T. Kasai y J. Kawabata, Nutritional evaluation of wild yam (Dioscorea spp.) Tubers of Nepal, Journal Food Chemistry, 82(4), 619-623 (2003)

Casada, M. y otros tres autores, Moisture content as a function of temperature rise under microwave radiation, Journal Transactions of the ASAE, 14(2), 907-911 (1983)

Click, L. y C. Baker, Moisture determinations of agricultural products using a microwave oven, ASAE Paper, 80-3050 (1980)

Duffau, B. y otros siete autores, Validación de métodos y determinación de la incertidumbre de la medición: "Aspectos generales sobre la validación de métodos". Instituto Salud Publica Chile. Santiago. 21-53 (2010)

Espinosa, Y., Influencia de las condiciones de extracción sobre el rendimiento del proceso de lixiviación de almidón en tres clones de ñame pertenecientes a la especie Dioscorea alata (0506-112. 9303-036. 9605055).Tesis Ingeniero de Alimentos. Universidad de Córdoba. Córdoba (2009)

Gallina, A., N. Stocco y A. Mutinelli, Titration to determine moisture in honey: a new simplified approach, Food Control, 21, 942-944 (2010)

García-Mogollón, C., S. Dussán-Sarria y N. Gutiérrez-Guzmán, Uso de horno microondas en la determinación de contenido de humedad: yuca, ñame y plátano, Revista Biotecnología en el Sector Agropecuario y Agroindustrial, 10(1), 60-66 (2012)

Gorakhurwalla, H., R. Ginty y C. Watson, Determining moisture content of grain using microwave energy for drying, Journal Agricultural Enginnering Resource, 20(3), 319-325 (1975)

Hall, B., Single Laboratory Validation. Official Methods of Analysis, AOAC International (2008)

Kraszewski, A., S. Nelson y T. You, Moisture content determination in single corn kernels by microwave resonator techniques, Journal Agricultural Enginnering Research, 48(1), 77-87 (1991)

Kress-Rogers, E. y M. Kent, Microwave measurement of powder moisture and density, Journal of Food Engennering, 6(5), 345-376 (1987) 
Martínez, D. y I. Ortiz, Evaluación de las propiedades tecnofuncionales de los almidones de ñame a partir de tres clones de la especie Discorea rotundata (9811-083. 9811-089. 9811-091). Tesis Ingeniero de Alimentos. Universidad de Córdoba. Córdoba (2009)

Mantanus, J. y otros siete autores, Moisture content determination of pharmaceutical pellets by near infrared spectroscopy: method development and validation, Anal Chm Acta, 642(1-2), 186-192 (2009)

Nyfors, E., Industrial Microwave Sensors - A Review, Journal Subsurface Sensing Technologies and Applications, 1(1), 23-43 (2000)

Oetzel, G., F. Villalba, W. Goodger y K. Norlund, Comparison of on-farm methods for estimating the dry matter content of feed ingredients, Journal of Dairy Science, 76(1), 293-299 (1993)

Reina, Y., El cultivo del ñame en Colombia, Documentos de Trabajo sobre Economía Regional, Centro de Estudios Regionales, CEER, 12(168), 2-21 (2012)

Romero, F. y J. Jiménez, Evaluación de la cinética de secado y rehidratación de chips de ñame (Dioscorea rotundata) secadas en horno microondas. Tesis Ingeniero de Alimentos. Universidad de Córdoba. Córdoba (2014)

Sorland, G. y otros cuatro autores, Determination of total fat and moisture content in meat using low field NMR, doi: 10.1016/S0309-1740(03)00157-8, Meat Science, 66(3), 543-550 (2004)

Tirado, D., P. Montero y D. Acevedo, Estudio comparativo de métodos empleados para la determinación de humedad de varias matrices alimentarias, Información Tecnológica, 26(2), 3-10 (2015)

Zumbado, H., Análisis Químicos de los Alimentos. Métodos Clásicos, Instituto de Farmacia y Alimentos, Universidad de la Habana, Habana, Cuba (2004)

Mantanus, J. y otros siete autores, Moisture content determination of pharmaceutical pellets by near infrared spectroscopy: method development and validation, doi: 10.1016/j.aca.2008.12.031, Analytica Chimica Acta, 642 (1-2), 186-192 (2009)

Sorland, G. y otros cuatro autores, Determination of total fat and moisture content in meat using low field NMR, Meat Science, 66, 543-550 (2004)

Gallina, A., N. Stocco, y F. Mutinelli, Karl Fischer Titration to determine moisture in honey: A new simplified approach, Food Control, 21(6), 942-944 (2010)

Casada, M.E., L.R. Walton, L.D. Swetnam, y J.H. Casada, Moisture content as a function of temperature rise under microwave radiation, Transactions of the ASAE, 26(3), 907-911 (1983) 
